23 HIDDEN: A HOSPICE INPATIENT DEEP VEIN THROMBOSIS DETECTION STUDY TO EXPLORE THE PREVALENCE, SYMPTOM BURDEN AND NATURAL HISTORY OF VENOUS THROMBOEMBOLISM IN PEOPLE WITH ADVANCED CANCER

MJ Johnson, SIR Noble, F Swan, V Allgar, E Napier, A Nelson, M Watson, C White. University of Hull, University of Cardiff, University of York, Belfast NHS Trust, Hospice UK, Northern Ireland Hospice

\subsection{6/bmjspcare-2019-ASP.22}

Background The deep venous thrombosis (DVT) prevalence in advanced cancer is unconfirmed and it is unknown whether current international thromboprophylaxis guidance is applicable to this group. We determined prevalence and predictors of femoral DVT in patients admitted to specialist palliative care units (SPCU).

Methods Prospective longitudinal observational study in five SPCUs in England, Wales and Northern Ireland. Consecutive adults with cancer underwent bilateral femoral vein ultrasonography on admission and weekly until death or discharger for a maximum of three weeks. Data were collected on performance status, attributable symptoms and variable known to be associated with venous thromboembolism. Patients were ineligible if admitted for terminal care (estimated prognosis < five days). Prevalence was estimated with 95\% confidence intervals (CI). DVT predictors and survival were explored using regression analyses. Sensitivity analysis excluded early scans to account for a technical learning curve.

Results 343 participants (68.2 [SD 12.8] 25 to 102 years; men 52\%; AKPS 49 [SD 16.6] 20\% to 90\%) were recruited. Of 273 evaluable scans, 92 (34\%, CI 28\% to 40\%) showed DVT. Excluding early scans, 64/232 (28\%, 22\% to 34\%) showed DVT. Four participants with a 'no DVT' scan on admission developed a DVT on repeat scanning over 21 days. Previous thromboembolism, bedbound $\leq 12$ weeks for any reason $(p=0.003)$ and lower limb oedema $(p=0.009)$ independently predicted DVT. Serum albumin $(p=0.430)$, thromboprophylaxis $(p=0.173)$ and survival $(p=0.473)$ were unrelated to DVT.

Conclusions These novel data show approximately one third of SPCU admissions with advanced cancer had a femoral DVT. DVT was not associated with thromboprophylaxis, survival or symptoms other than leg oedema. Findings are consistent with VTE being a manifestation of advanced disease rather than a cause of premature death. Thromboprophylaxis for SPCU inpatients with poor performance status seems of little benefit.

\section{EXPERIENCES OF OLDEST-OLD CARERS WHOSE PARTNER IS APPROACHING END-OF-LIFE: A SYSTEMATIC REVIEW AND NARRATIVE SYNTHESIS}

Tessa Morgan, Aamena Bharmal, Robbie Duschinsky, Stephen Barclay. University of Cambridge

\subsection{6/bmjspcare-2019-ASP.23}

Background Globally ageing populations have rapidly increased the number of people requiring palliative care. In resourcelimited healthcare systems, spouses are increasingly providing end-of-life care to enable their partners to remain at home. Little attention, however, has been paid to oldest-old spousal carers who are often managing their own co-morbidities whilst caring.
Aim To review the literature concerning experiences of carers aged 75 and over whose partner is approaching end-of-life.

Methods Systematic review and narrative synthesis of the empirical literature published between 1985-April 2018 across six databases. Gough's weight of evidence and Morgan's feminist appraisal tool used to determine quality of papers.

Results From the initial 6950 titles, eight qualitative studies and six quantitative studies were included. Five studies focused exclusively on care recipients with severe dementia. We identified three themes. (1) 'The embodied impact of care'. Many oldest-old carers experienced a 'double jeopardy' while providing emotionally and physically difficult end-of-life care and managing their own diminishing health. They often subordinated their own health needs to continue caring. (2) 'Marital and gendered conceptualisations of caring'. Oldest-old carers strove for normality in their self and marriage identities by providing care for their spouse with little additional support and by incorporating their caregiving within their established gender roles. (3) 'Learning to care'. Oldest-old carers had to learn new skills and ways of coping to keep their spouses at home. They became the expert of their partners' condition as well as having to take over all household tasks.

Conclusion This review highlights the importance of oldest-old carers contributions to their spouses' end-of-life care. Oldestold carers see it as their duty to care, but the caregiving itself involves learning new practical and emotional skills. Future research needs to consider how to best support these carers to do so without grave impact to their own health.

\section{Poster presentations}

\section{Bereavement | Posters 1 - 3}

\section{RETURNING TO WORK IN SPECIALIST PALLIATIVE CARE FOLLOWING PERSONAL BEREAVEMENT}

Paul Davies, Adam Hurlow, Suzie Gillon. Leeds Teaching Hospitals NHS Trust

\subsection{6/bmjspcare-2019-ASP.24}

Background During their working career, most people will experience a personal bereavement. Our perception was that a return to work might be difficult in a speciality with high patient mortality rates. Despite this, there is a paucity of evidence concerning the impact of bereavement on palliative care professionals. Staff recruitment and retention remains an ongoing challenge requiring optimisation of workforce support.

This study sought to elicit the factors that affect palliative care team-members' return to work following personal bereavement.

Methods An anonymised questionnaire based survey was distributed to specialist palliative care teams in Yorkshire for completion by clinical staff with patient contact.

Responses were analysed thematically.

Results 408 responses were returned from 857 questionnaires distributed (48\% response rate), of which 257 staff members had experienced personal bereavement whilst working in palliative care. Of the 169 who had absence from work: $35 \%$ were absent $\leq 1$ week; $46 \%>1$ week and $\leq 4$ weeks; $12 \%>4$ weeks $\leq 8$ weeks, and $6.5 \%>8$ weeks. A number of staff found returning to work challenging. There was significant variation in perceived support from colleagues; managers and organisations. 
Broad areas workers found helpful were good communication from managers, flexibility of duties on return to work (including temporary non-clinical roles), sympathetic support from colleagues and access to counselling support from organisations.

Broad areas workers found unhelpful were an absence of meetings and communication from managers, rigid compassionate leave policies and perceived lack of recognition of their loss.

Conclusions There was variation between individuals about factors they perceived to be helpful. This suggests a rigid approach universally applied is unlikely to support all employees. In our opinion, early sympathetic meetings with management offering temporary adjustments to roles (and/or hours), sensitive informing of colleagues and signposting to counselling options is likely to have a positive impact on staff wellbeing.

\section{MAPPING OF BEREAVEMENT SERVICES ACROSS THE NORTH EAST OF ENGLAND - A SURVEY OF GENERAL PRACTITIONERS REFERRING PRACTICE}

D Wakefield, F Dewhurst, J Brown, E Kavanagh, R Kiltie, F McCormick, J Pickard, G Rowley, K Waterfield, L Woods, A Clark. Palliative Medicine Registrars, Health Education North East (HENE)

\subsection{6/bmjspcare-2019-ASP.25}

Background Recent national guidelines have identified the importance of bereavement support. However, evidence suggests that bereavement services are provided inconsistently. We aimed to map bereavement services used by GPs across the North-East-of-England to identify inequalities.

Method An electronic bereavement service questionnaire was produced through consultation with Northern England Clinical Networks and CCG end-of-life-care leads and was then reviewed by a local GP focus group. The questionnaire was then distributed to CCG EOLC leads who cascaded it to all 392 practices in the North-east of England. A Reminder email was sent after 4 weeks.

Results 85 (22\%) GP practices completed the survey. Only $43.5 \%$ and $9.4 \%$ of practices reported that they had a bereavement policy and that the majority of their GPs' had had bereavement training respectively. $21 \%$ reported that they would not refer patients anywhere for bereavement support. The remaining 79\% listed 18 services that they refer to, most commonly; hospices(21\%), CRUSE(17\%), MIND(15\%), Macmillan(10\%) and their own in-house counsellor(10\%) and 25 services that they recommend for self-referral, CRUSE(65\%), talking therapy(16\%), hospice(14\%), Improved Access to Psychological Therapies(11\%).

Conclusion A wide range of bereavement services are used across the region. They mainly depended on charitable funding. One fifth of responding GP practices reported that they would not refer patients for bereavement support. This was associated with comments including "local services have been decommissioned" and "not aware of any services to refer to". This highlights the wide variation in use and availability of bereavement services across the region, and the problems of service withdrawal, lack of funding and lack of clarity on who is responsible for bereavement support. This will be invaluable in providing evidence to commission future bereavement services.

\section{INNOVATIVE FOUR STEP BEREAVEMENT SERVICE AT A TERTIARY CANCER CENTRE}

Ann Griffiths, Alison Coackley, Malcolm Cooper, Amanda Watson, Sinead Benson, Claire Cadwallader, Dan Monnery, Julian Hampton-Matthews. The Clatterbridge Cancer Centre

10.1136/bmjspcare-2019-ASP.26

Background Bereavement is defined as the state of a loss when someone close to you has died. It is important that people closely affected by a death are communicated with in a sensitive and timely manner and that those at risk of pathological bereavement reactions are identified and supported. At a tertiary cancer centre it was recognised that families need time and support soon after the death of their loved one.

Aim To deliver a 'day after death' service providing personalised bereavement support to families/carers and learn lessons about care by discussing their experiences.

Methods At our tertiary cancer centre we implemented an innovative 4 step approach to supporting bereaved families and carers:

1. Comprehensive documentation including a bereavement risk assessment at the time of death.

2. Innovative 'day after death' service.

3. Post bereavement contact if accepted.

4. Face to face follow up where required.

The bereaved families/carers meet with a senior member of the Nursing Team who knew the patient on the next working day. They meet in a private room away from the wards and discuss how they are coping with their loss and listen to any questions/concerns they may have. The relevant paperwork is then provided. This also provides the opportunity to follow up the bereavement risk assessment undertaken by the ward staff.

Following the meeting a reports is shared with the Director of Nursing and discussed at the Trust Executive meeting.

Results There have been 84 deaths within the tertiary cancer centre in the last 12 months. All bereaved families/carers have received the day after death service. This has enabled us to provide comprehensive and personalised bereavement care. It also enables us to identify those people likely to have complex bereavement and signpost to local specialist support services. Conclusions We have successfully implemented a 4 step innovative service delivering personalised bereavement care.

\section{Caregivers and the Family | Posters $4-7$}

\section{TYPE, INTENSITY AND QUALITY OF INFORMATION GIVEN BY PHYSICIANS DURING THE CANCER JOURNEY OF PALESTINIAN CHILDREN WITH LEUKAEMIA: BETWEEN REVELATION AND CONCEALMENT}

Maha Atout, Bernie Carter. Philadelphia University, Edge Hill University

\subsection{6/bmjspcare-2019-ASP.27}

Background Despite the accumulation of evidence that supports the importance of giving parents detailed information, there is less evidence to support the notion that providing 'negative' information has a harmful effect on both parents and children. This study explored the communication of information across the cancer journeys taken by children from 